\title{
REGULAR ELEMENTS IN SEMIPRIME RINGS
}

\author{
R. E. JOHNSON AND L. S. LEVY
}

In the proof of Goldie's theorem [1, Theorem 4.1], one of the crucial steps is to establish that every large right ideal contains a regular element [1, Theorem 3.9]. Recently, S. A. Amitsur told one of the authors he had proved, using the weaker conditions of the ACC on left and right annihilators, that every prime ring contains a left regular element $a$ (i.e., the left annihilator $a^{l}$ of $a$ is zero) and a right regular element $b$ (i.e. the right annihilator $b^{r}$ of $b$ is zero). In this note, we generalize Amitsur's result as follows:

THEOREM. In a semiprime ring $R$ with $A C C$ on left and right annihilators, every large right ideal contains a regular element.

We remark that for semiprime rings, Goldie's conditions on a ring imply ours [5, Lemma 2]. However, the converse does not hold, since there exist noncommutative integral domains which do not have a right quotient division ring (see, e.g. $[1, \S 6]$ ).

We also remark that although the ring $R$ in our theorem need not have a (classical) right quotient ring, our result shows that $R$ does have a maximal right quotient ring $Q$ (in the sense of Johnson) and for each $q \in Q$ there exist $d, r \in R$, with $d$ regular, such that $q d=r$.

To remove any doubt about terminology, the ACC on right annihilators means that the ACC holds in the set of all right ideals of the form $\{z \in R \mid S z=0\}, S$ any subset of $R$, and a right ideal is large if it intersects every nonzero right ideal nontrivially. The notation $Z_{r}(R)$ is used for the right singular ideal of $R: Z_{r}(R)=\left\{a \in R \mid a^{r}\right.$ large $\}$.

LEMMA. (i) If a prime ring $R$ has a maximal right annihilator then $Z_{r}(R)=0$. (ii) If $R$ is a ring having $Z_{r}(R)=0$ and the $A C C$ on right annihilators, then $a^{r}=0$ whenever $a R$ is a large right ideal of $R$.

Part (i) is proved in [3, Theorem 1], whereas part (ii) is proved in [2, Theorem 3.3].

Proof of The theorem. We note that the ACC on right annihilators is equivalent to the DCC on left annihilators. We first prove the theorem in the special case that $R$ is prime.

Let $A$ be a large right ideal of the prime ring $R$. We shall show that for every $a \in A$ such that either $a^{r} \neq 0$ or $a^{l} \neq 0$, there exists $b \in A$

Received by the editors October 11, 1966 and, in revised form, April 21, 1967. 
such that $b^{r} \subseteq a^{r}$ and $b^{l} \subseteq a^{l}$ with at least one of these inequalities strict. By the DCC on right and left annihilators, it will follow that $a^{r}=0$ and $a^{l}=0$ for some $a \in A$.

By Zorn's lemma, $R$ contains direct sums of the form $a R \oplus B$, $B \subset A$, and $R a \oplus C$ ( $B$ and $C$ being right and left ideals of $R$, respectively), where $a R \oplus B$ is a large right ideal of $R$ and $R a \oplus C$ is a large left ideal.

If $a^{r} \neq 0$, then by the lemma (and its left-handed version) neither $a R$ nor $R a$ is large in $R$; hence $B$ and $C$ are nonzero. The same conclusion holds if $a^{l} \neq 0$. Since $R$ is prime, $B C \neq 0(B C \subseteq A)$ and we have the following nontrivial direct sums of additive groups:

$$
a R \oplus B C \subseteq a R \oplus B, \quad R a \oplus B C \subseteq R a \oplus C .
$$

The directness of the sums in (1) shows that for every $y \in B C$

$$
(a+y)^{r} \subseteq a^{r}, \quad(a+y)^{l} \subseteq a^{l} .
$$

We claim that if $a^{r} \neq 0$, then the first inequality of (2) is strict for some $y \in B C$. For if $(a+y)^{r}=a^{r}$ for every $y \in B C$, then $B C a^{r}=0$. Since $R$ is prime and $B$ is a right ideal, necessarily $C a^{r}=0$ and hence $(R a \oplus C) a^{r}=0$. However, this equation contradicts the fact, according to the lemma, that $Z_{l}(R)=0$.

Similarly, if $a^{l} \neq 0$, there exists some $y \in B C$ such that the second inequality of (2) is strict. Thus, the theorem is proved if $R$ is prime.

We now carry out a reduction to the prime case, making use of the theorem [4, Theorem 3.13] that a semiprime ring $R$ satisfying the ACC on two-sided annihilator ideals is an irredundant subdirect sum of a finite number of prime rings. That is, there exist prime rings $R_{1}, \cdots, R_{n}$ whose direct sum contains $R$, such that $p_{i}(R)=R_{i}$ for each coordinate map $p_{i}: R \rightarrow R_{i}$, and

$$
R \cap R_{i} \neq\{0\}, \quad i=1 \cdots, n .
$$

The ideals ker $p_{i}$ are the maximal two-sided annihilator ideals of $R$ [4, Theorem 3.2].

Another fact which we will need is that if $R$ has the ACC on left and right annihilators, then each $R_{i}$ satisfies the same conditions. To prove this, we first observe that each two-sided ideal $I$ of $R$ which is an annihilator right ideal is also an annihilator left ideal. Next, we observe that the inverse image $B$ in $R$ of each annihilator right (left) ideal $\bar{B}$ in $\bar{R}=R / I$ is an annihilator right (left) ideal of $R$. Thus, if $I=X^{r}$ and $\bar{B}=\bar{Y}^{r}$ then $B=(X Y)^{r}$, and similarly for left annihilators. Consequently, each $R_{i} \cong R / \operatorname{ker} p_{i}$ has the $\mathrm{ACC}$ on left and right annihilators. 
Now let $A$ be a large right ideal of $R$ and let $A_{i}=p_{i}(A)$. We show that $A \cap A_{i}$ is a large right ideal of $R_{i}$. If $B_{i}$ is a nonzero right ideal of $R_{i}$, then $B_{i}\left(R \cap R_{i}\right)$ is a nonzero right ideal of both $R$ and $R_{i}$ since $R$ is prime and $R \cap R_{i} \neq 0$. Since $A$ is large in $R, A$ meets $B_{i}\left(R \cap R_{i}\right)$ nontrivially. But every element of this "meet" belongs to $A$ and $B_{i}$, hence to $R_{i}$, and hence to $A_{i}$. Thus $A \cap A_{i}$ meets $B_{i}$ as desired.

Now, by the prime case of the theorem, each $A \cap A_{i}$ contains a regular element $a_{i}$ of $R_{i}$. The element $a_{1}+a_{2}+\cdots+a_{n}$ of $A$ is thus regular in $R$, and the proof of the theorem is complete.

\section{BIBLIOGRAPHY}

1. A. W. Goldie, Semi-prime rings with maximum condition, Proc. London Math. Soc. (3) 10 (1960), 201-220.

2. R. E. Johnson and E. T. Wong, Quasi-injective modules and irreducible rings, J. London Math. Soc. 36 (1961), 260-268.

3. K. Koh and A. C. Mewborn, Prime rings with maximal annihilator and maximal complement right ideals, Proc. Amer. Math. Soc. 16 (1965), 1073-1076.

4. L. S. Levy, Unique subdirect sums of prime rings, Trans. Amer. Math. Soc. 106 (1963), 64-76.

5. C. Procesi and L. Small, On a theorem of Goldie, J. Algebra 2 (1965), 80-84.

UNIVERSITY OF NEW HAMPSHIRE AND

UNIVERSITY OF WISCONSIN 